\title{
Temporal epigenetic modifications differentially regulate ES cell-like colony formation and maturation
}

\author{
Jong S. Rim ${ }^{1^{*}}$, Karen L. Strickler ${ }^{1^{*}}$, Christian W. Barnes ${ }^{1}$, Lettie L. Harkins ${ }^{1}$, \\ Jaroslaw Staszkiewicz ${ }^{1}$, Jeffrey M. Gimble ${ }^{2}$, Gregory H. Leno ${ }^{3}$, Kenneth J. Eilertsen ${ }^{1,2^{*}}$ \\ ${ }^{1}$ NuPotential Inc., Baton Rouge, USA; ${ }^{2}$ Corresponding Authors: jsrnupotential@laetc.com, kjenupotential@laetc.com \\ ${ }^{2}$ Stem Cell Biology Laboratory, Pennington Biomedical Research Center, Baton Rouge, USA \\ ${ }^{3}$ Department of Anatomy \& Cell Biology, Carver College of Medicine, University of Iowa, Iowa City, USA
}

Received 8 February 2012; revised 1 March 2012; accepted 13 April 2012

\begin{abstract}
Human somatic cells can be directly reprogrammed to induced pluripotent stem (iPS) cells by forced expression of the transcription factors Oct4, Sox2, and either KIf4 and cMyc or Nanog and Lin28, using virus-based systems. However, low reprogramming efficiency and the potential for deleterious virus-induced genomic modification limit the clinical potential of this technology. Recent reports indicate, however, that the generation of iPS cells can be enhanced by the addition of synthetic small molecules, including epigenetic modulators. In this report, we demonstrate that the epigenetic modifiers Valproic Acid (VPA) and 5-azacytidine activate the reciprocal transcriptional regulation of endogenous pluripotency transcription factor genes in human dermal fibroblasts and that VPA alone can directly activate endogenous Oct4 in the absence of transgenes. Moreover, using human adipose cells, we demonstrate that histone deacetylase inhibition, prior to reprogramming factor transfection, increases embryonic stem (ES) cell-like colony formation $\sim 2$ - 3 fold. In addition, DNA methyltransferase (DNMT) inhibition during human ES cell culture promotes maturation of reprogrammed somatic cells, increasing the yield $\sim 4$ fold. These data provide proof of principle that reprogramming efficiency can be improved by inhibiting specific repressive epigenetic regulatory components at the levels of ES cell-like colony formation and maturation. In addition, these studies raise the interesting possibility that a more efficient small molecule-based reprogramming system may provide a superior alternative to current virus-based approaches.
\end{abstract}

Keywords: Epigenetic Modification; Somatic Cell Reprogramming

\section{INTRODUCTION}

Forced expression of the transcription factors Oct4, Sox2, and either Klf4 and cMyc or Nanog and Lin28 can transform differentiated somatic cells into induced pluripotent stem (iPS) cells. In many respects, iPS cells are the functional equivalent of pluripotent embryonic stem (ES) cells [1-3] and therefore, hold enormous research and clinical potential. For example, iPS cells may facilitate mechanistic studies for degenerative diseases, drug discovery and toxicity testing. Indeed, several reports have described and validated "disease-in-a-dish" model systems [4-8]. The prospect for clinical application is limited, however, by reprogramming inefficiency and safety issues associated with viral vector transduction.

The efficiency of iPS cell production is influenced by the reprogramming strategy employed. Indeed, the number and combinations of transcription factors, the origin and developmental stage of the recipient cell, the method of transduction, and the use of small molecule epigenetic modifiers all play a role in determining the degree of iPS cell formation and colony maturation. Several general conclusions can be drawn from these studies. First, expression of pluripotency-related transcription factors while silencing p53 improves reprogramming efficiency $[9,10]$. Second, somatic cells from early development stages have greater reprogramming potential than mature cells [2]. Third, more efficient transfection methods, such as the polycistronic lentiviral system, improves reprogramming [11]. Finally, exposure to small molecule epigenetic modifiers also increases iPS cell production [12].

Reprogramming strategies are also being developed that address the safety concerns raised by the widely used virus-based reprogramming platforms. These include the use of non-integrating plasmids and mRNA [10,13-15], miRNA [16], excisable transposons [17,18], 
and proteins $[19,20]$. Identification of a small molecule or a cocktail of small molecules, to reprogram cells also promises to provide safer iPS cells and enhance opportunities for scalability ([21-24] see review, [25]).

Although the molecular mechanisms involved in somatic cell reprogramming are not well defined, it is clear that epigenetic modification has proven to be critical for de-differentiation of somatic cells. The most well studied example is promoter specific DNA de-methylation and histone acetylation. These changes are required for the re-expression of pluripotency-related genes that are silenced during animal development [26]. Recent genomewide mapping of target promoters for Oct4, Sox2, Klf4 and c-myc, as well as Nanog, Dax1, Rex1, Zpf281, and Nac1 factors, has provided a more detailed and global understanding of the pluripotent epigenome in mouse ES cells [27]. Among the more than 6000 target gene promoters analyzed, two categories emerged with respect to transcription factor binding and promoter activity. First, target promoters bound by four or fewer factors are repressed while those associated with a higher factor density are active. Remarkably, the transcriptional network of ES cells includes auto- and reciprocal-transcriptional regulation as well as protein-protein interactions that contribute to regulate pluripotency gene expression.

We hypothesize that epigenetic modification directly activates an endogenous pluripotent transcription network resulting in the increased efficiency of transcription factor-based somatic cell reprogramming. In this report, we used two epigenetic modifying agents, an inhibitor of DNA methyltransferase (DNMT) and a histone deacetylase (HDAC) inhibitor, to begin to test this hypothesis. We found that both DNMT and HDAC inhibitors activate the endogenous reprogramming factor transcription network in human dermal fibroblasts. In addition, we demonstrated that exposure of human preadipocytes to an HDAC inhibitor, prior to transfection, increases the induction of initial ES-like colony formation and that exposure to a DNMT inhibitor after transfection, facilitates iPS colony maturation.

\section{MATERIALS AND METHODS}

\subsection{Cell Culture}

Human dermal fibroblasts from adult, neonatal and fetal origin (Cell Applications Inc., San Diego, CA) were cultured with fibroblast growth medium (Cell Applications) at $37^{\circ} \mathrm{C}, 5 \% \mathrm{CO}_{2}$ in a humidified incubator. Human preadipocytes (Cell Applications) were maintained with preadipocyte growth medium (Cell Applications) or Dulbecco's modified Eagle medium (DMEM) supplemented with $10 \%$ FBS, $50 \mathrm{U} / \mathrm{ml}$ penicillin and $50 \mu \mathrm{g} / \mathrm{ml}$ streptomycin at $37^{\circ} \mathrm{C}, 5 \% \mathrm{CO}_{2}$ in a humidified incubator. Human adipose derived adult stem cells (Stem Cell Bi- ology Laboratory, Pennington Biomedical Research Center, Baton Rouge, LA) were maintained with DMEM supplemented with $10 \% \mathrm{FBS}, 50 \mathrm{U} / \mathrm{ml}$ penicillin and 50 $\mu \mathrm{g} / \mathrm{ml}$ streptomycin at $37^{\circ} \mathrm{C}, 5 \% \mathrm{CO}_{2}$ in a humidified incubator. The human adipose derived stem cells were obtained from subjects undergoing elective surgery with written informed consent under a protocol reviewed and approved by the Pennington Biomedical Research Center Institutional Review Board (PBRC \#23040). Cell culture medium was supplemented with $5 \mathrm{mM}$ VPA (Sigma), 5 $\mu \mathrm{M}$ 5-azacytidine (Sigma), $1 \mu \mathrm{M}$ TSA (Sigma), $5 \mu \mathrm{M}$ Scriptaid (BioMol) or $25-100 \mu \mathrm{M}$ Zebularine (Sigma, concentrations as indicated in the figure) for the treatment as indicated. Adipose cell derived iPS (AdiPS-Ctl, AdiPS-TSA) cells were generated from human preadipocytes (Cell Applications) or human adipose derived adult stem cells as described in the paper, and maintained on an irradiated mouse embryonic fibroblast (MEF) feeder layer from ATCC (Manassas, VA) or on Matrigel coated cell culture plates (BD Biosciences) with mTeSR1 hES culture medium (StemCell Technology). Mel-1 human ES cells were purchased from Millipore (Temecula, CA) and maintained on MEF feeder layer (ATCC, Manassas, VA) with mTeSR1 hES culture medium (StemCell Technology).

\subsection{Lentiviral Transduction and Generation of AdiPS Cells}

High titer lentivirus, overexpressing human Oct4 or Nanog proteins, was purchased from System Biosciences (San Diego, CA). The day before lentiviral transfection, human dermal fibroblasts were trypsinized, counted, and seeded in 6-well plates at a density of $4 \times 10^{5}$ cells/well. The next day, culture medium was replaced with prewarmed medium containing $3 \mu \mathrm{g} / \mathrm{ml}$ polybrene (Sigma) and 5 MOI of lentivirus. Eighteen hours later, culture medium was changed with fresh fibroblast growth medium or treated with histone deacetylase inhibitor (HDACi) or DNA methyltransferase inhibitor (DNMTi). After 7 days treatment, total RNA was isolated for gene expression analysis. High titer polycistronic lentivirus STEMCCA (Oct4, Sox2, Klf4, c-Myc) (Millipore, Temecula, CA) were transfected into human preadipocytes. Briefly, $10^{5}$ human preadipocytes that had been cultured with or without HDACi and/or DNMTi, were transfected with 75 MOI of STEMCCA lentivirus overnight. Lentiviral infection was repeated the next day, and culture medium was changed with fresh preadipocyte growh medium. When the cells reached confluence, they were trypsinized, counted and seeded on a prepared MEF feeder layer at a density of $5 \times 10^{4}$ cells with mTeSR1 medium (StemCell Technology) supplemented with $10 \mu \mathrm{M}$ ROCK inhibitor (ROCKi) (Stemgent). 


\subsection{Quantitative Realtime RT-PCR}

Total RNA was prepared from cultures using Trizol Reagent (Life Technology) and the RNeasy Mini RNA isolation kit (Qiagen) with DNase I digestion. Total RNA $(1 \mu \mathrm{g})$ from each sample was subjected to oligo (dT)primed reverse transcription (Invitrogen) to generate cDNA. Quantitative PCR to measure mRNA expression levels was performed with Taqman gene expression assays (Applied Biosystems) using a 7300 real-time PCR system in the genomic core facility at Pennington Biomedical Research Center. Expression levels were compared to known standard samples and were normalized to GAPDH.

\subsection{Immunofluorescence and Alkaline Phosphatase Staining}

Cells were fixed with 4\% paraformaldehyde in PBS for $10 \mathrm{~min}$, and incubated for $1 \mathrm{hr}$ with antibodies specific for pluripotency markers Oct4, Nanog, Sox2 (Abcam) and TRA1-60 (Millipore). After washing three times with PBS, cells were incubated for $1 \mathrm{hr}$ with fluorescent conjugated secondary antibody (Invitrogen). Nuclei were detected by DAPI staining (Vector shield). Alkaline Phosphatase staining was performed using the Alkaline Phosphatase Staining kit (Stemgent).

\subsection{In Vitro Differentiation}

Human AdiPS cells cultured on MEF feeder layers were treated with dispase (StemCell Technology) and transferred to Matrigel coated plates (BD Biosciences) with mTeSR1 culture medium. When attached AdiPS colonies started to contact each other, spontaneous differentiation was initiated by changing medium with Dulbecco's modified Eagle medium (DMEM) supplemented with $10 \%$ FBS, $50 \mathrm{U} / \mathrm{ml}$ penicillin and $50 \mathrm{ug} / \mathrm{ml}$ streptomycin. Continuously beating (heart) cells were observed by microscopy.

\subsection{Teratoma Formation}

For in vivo teratoma formation, human AdiPS cells were collected with cell recovery solution (BD Biosciences). After washing with ice cold PBS, cells were resuspended at $4 \times 10^{6}$ cells $/ \mathrm{ml}$ in PBS and mixed with BD matrigel matrix $\mathrm{HC}$ (BD Biosciences) in a final volume of $0.5 \mathrm{ml}$ on ice for each injection. Cell suspensions were injected subcutaneously in athymic nude mice using a $23 \mathrm{G}$ needle. Six weeks after the injection, tumors were surgically dissected and each teratoma was evaluated by H\&E staining. Experiments involving vertebrate animals were approved by Pennington Biomedical Research Center's Institutional Animal Care and Use Committee (A3677-01, protocol \#624) and conformed to or exceeded NIH stan- dards.

\subsection{Bisulfite Sequencing}

DNA was isolated and purified by DNeasy Blood and Tissue kit (Qiagen). Bisulfite conversion was performed using the EZ DNA Methylation kit (Zymo Research). Converted DNA was amplified by PCR using primers for Oct3/4 or Nanog as follows: Oct4 forward primer: 5'-GTTAGAGGTTAAGGTTAGTGGGTG Oct4 reverse primer: 5'-AAACCTTAAAAACTTAACCAAATCC

Nanog forward primer: 5'-TGGTTAGGTTGGTTTTAAATTTTTG

Nanog reverse primer: 5'-AACCCACCCTTATAAATTCTCAATTA

Dlk1 \#1 forward primer: 5'-GTTTTTTTGTTTTTGTTGGTTTT

Dlk1 \#1 reverse primer: 5'-ACTAAAAATCTCACACATCCCCTAC

Dlk1 \#2 forward primer: 5'-AGTTGTATTTGGGTGAATGGATTAT

Dlk1 \#2 reverse primer: 5'-AAAACAAAAAAACCAAACAAAAAAC

Dlk1 \#3 forward primer: 5'-TTTAATAGGAGAGGGTGGAGATGTA

Dlk1 \#3 reverse primer: 5'-CCTTACCTAAAATCACAAATCAAAAA.

PCR products were cloned into $E$. coli using the TOPO TA cloning kit (Invitrogen). Ten clones of each sample were verified by sequencing with SP6 and T7 primers.

\subsection{Low Density Microarray}

Human TaqMan ${ }^{\circledR}$ low density stem cell pluripotency arrays (TLDA) which contain 96 embryonic stem cell and post-implantation/differentiated tissue genes, and endogenous control genes were purchase from ABI (Applied Biosystems, Foster City, CA). Total RNA was isolated using an RNeasy RNA Isolation Kit (Qiagen, Valencia, CA), qualified, and quantified by spectrometry, and 50 ng total RNA was reverse transcribed to cDNA using the High Capacity cDNA Archive Kit (Applied Biosystems). TLDA qRT-PCR was performed as follows: for each sample, $100 \mu \mathrm{l}$ of the sample-specific PCR mix (including $5 \mu \mathrm{l}$ cDNA) was pipetted into the sample wells of the Micro Fluidic Card. The card was centrifuged, sealed, and run on the Applied Biosystem's 7900HT Sequence Detection System using the manufacturer's SDS 2.3 software. Relative quantification, using the $\Delta \Delta \mathrm{C}_{\mathrm{T}}$ method, was performed to determine fold changes in gene expression, relative to endogenous control, in reprogrammed stem cells compared to a baseline. 


\section{RESULTS}

\subsection{Activation of the Endogenous Reprogramming Factor Network by Inhibition of Histone Deacetylase or DNA Methyltransferase}

Differentiation silences pluripotency gene transcription during development and thereby, limits the genomic potential of the cell. This is partly due to epigenetic modifications of promoter regions by DNA methylation and histone modification. Thus, it stands to reason that the acquisition of pluripotency during cell reprogramming should also involve epigenetic modifications that enable pluripotency gene expression. To test this hypothesis, we first determined whether or not treatment of adult human dermal fibroblasts (HDFa) with epigenetic modulators increased expression of the pluripotency gene, Oct 4 . We found that increased mRNA expression of the endogenous Oct4 gene occurred after only three days of treatment with the histone deacetylase (HDAC) inhibitor, valproic acid (VPA, $5 \mathrm{mM}$ ), and to a lesser extent, with the DNA methyltransferase (DNMT) inhibitor, zebularine (Figure 1(a), $25 \mu \mathrm{M}, 50 \mu \mathrm{M}, 100 \mu \mathrm{M}$ ). The effect of zebularine on expression was concentration dependent. Surprisingly, however, differences in expression between control and zebularine-treated samples were not significant until day 7 of the incubation at a zebularine concentration of $100 \mathrm{mM}$ (Figure 1(a)). Oct4 mRNA levels remained elevated through 7 days of treatment with either inhibitor. However, expression levels of the endogenous Oct4 gene were much lower than the levels of ectopic expression achieved following transduction of HDFa cultures with a lentivirus expressing the human Oct4 gene (Figure 1(b)).

To determine the effects of VPA, and a second DNMT inhibitor, 5-azacytidine (5-aza), on ectopic expression of Oct4 and Nanog genes, we isolated mRNA from drugtreated HDFa cultures following Oct4 or Nanog lentivirus transduction. We then performed real-time RT-PCR using a gene specific primer and probes as described in Materials and Methods. We found that both VPA- and 5-aza-treated Oct4 lentivirus-infected cells had higher levels of Oct4 expression than that observed in control, VPA- or 5-aza-treated non-infected, and non-treated Oct4lentivirus infected cultures (Figure 1(c)). However, the absolute level of Oct4 mRNA expressed following induction was still one-third lower than that observed in human embryonic stem (hES) cells grown under standard conditions (data not shown). Results similar to those in HDFa cells were also observed in human dermal fibroblasts isolated from neonatal (HDFn) and fetal (HDFf) tissues.

This trend was also revealed with the transcription factor, Nanog. Specifically, VPA and 5-aza treatments each increased Nanog mRNA expression relative to control cultures (Figure 1(d)). In addition, VPA- and 5-azatreatment of cultures infected with Nanog lentivirus induced Nanog expression relative to control, VPA- or 5aza-treated non-infected, and untreated Nanog lentivirus infected cultures (Figure 1(d)). However, unlike Oct4 expression, the absolute level of Nanog expression was 10 -fold higher than the levels observed in hES cells. Taken together, these results suggest that the epigenetic modifiers, VPA and 5-azacytidine, activate the auto-transcriptional regulation of pluripotency transcription factors Oct4 and Nanog in somatic cells.

We also observed a slight induction of Oct4 (Figure 2(a)) and Nanog (Figure 2(b)) mRNA levels in Nanogand Oct4-lentivirus-infected HDFa cells, respectively, following VPA and 5-azaC treatments. Similarly, the low levels of endogenous Sox 2 that are normally observed in HDFf cells were increased by overexpression of Oct4 or Nanog and 5-azaC treatment (Figure 2(c)) However, VPA treatment did not significantly increase Sox 2 expression (Figure 2(c)). These data suggest that the epigenetic modifiers, VPA and 5-azaC, also activate the reciprocal transcriptional regulation of the endogenous pluripotency transcription factors in somatic cells.

\subsection{Induction of ES Cell-Like Colony Formation by Pre-Treatment of Human Preadipocytes with an HDAC Inhibitor}

The VPA- and 5-azaC-induced expression of Oct4 and Nanog genes raises the interesting possibility that epigenetic modification, prior to lentivirus transduction, may facilitate the reprogramming of somatic cells. Adult human adipose stem cells can be reprogrammed to iPS cells more rapidly and efficiently than human fetal skin fibroblasts [28] and therefore, we chose to use this system for our studies. Our experimental strategy is outlined in Figure 3(a). We first treated human pre-adipocytes with various HDAC inhibitors (HDACi) below their cell cycle inhibitory concentrations. Pre- (Day 7) and post-treated (Day 2) cells are shown in Figure 3(b) (left panel) and illustrate the change in cell density and morphology during HDACi treatment. These pre-treated cells were then transfected with lentivirus containing mouse Oct4, Sox2, Klf4 and cMyc cDNAs. Lentiviral transfection was repeated the next day and when the transfected cells reached confluence, they were split and transferred onto mouse embryonic fibroblast feeder layers. Colonies were initially apparent 9 days post-infection (Day 9) and each contained approximately $30-40$ cells that displayed typical hES morphology (Figure 3(b), right panel). By day 13 , tightly packed colonies with defined boundaries 


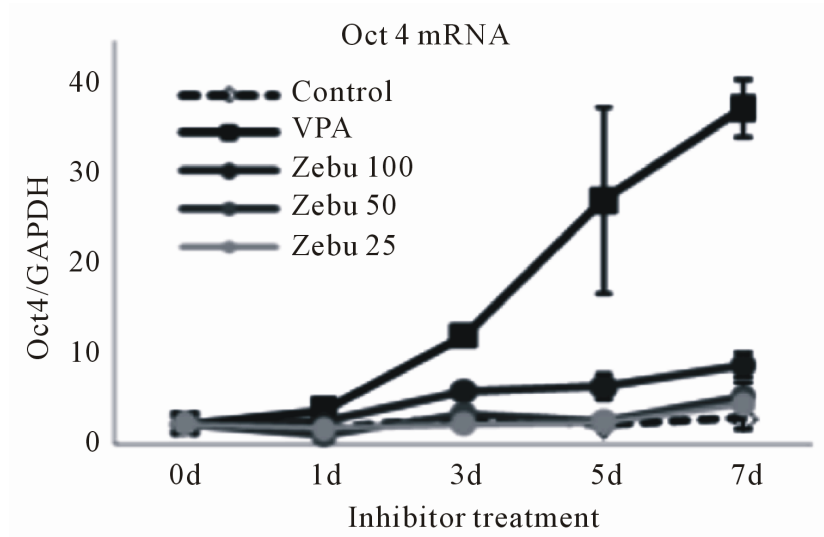

(a)

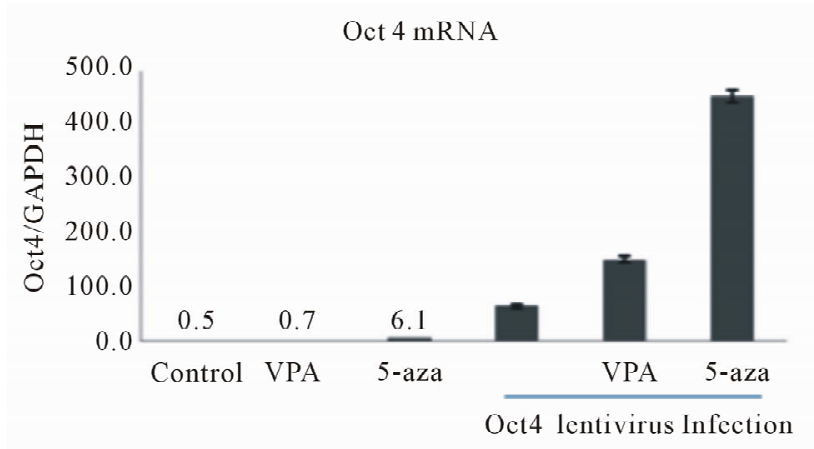

(c)

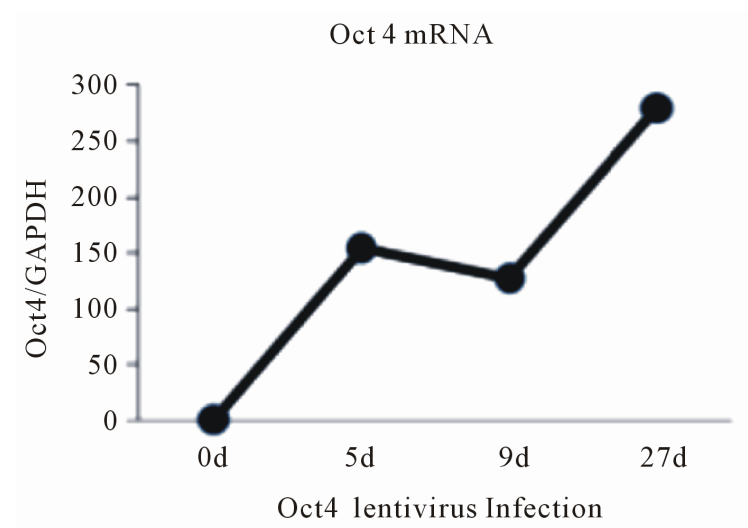

(b)

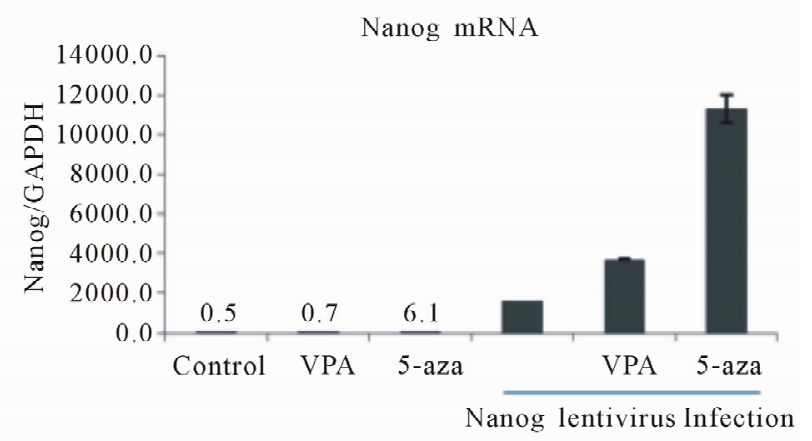

(d)

Figure 1. Induced expression of the Oct4 and Nanog genes in human dermal fibroblasts. (a) Analysis of Oct4 mRNA expression in adult human dermal fibroblasts (HDFa) following treatment with HDAC and DNMT inhibitors. Primary cultures from HDFa were treated with the HDAC inhibitor, Valproic acid (VPA, $5 \mathrm{mM})$, or the DNMT inhibitor, zebularine $(25 \mu \mathrm{M}, 50 \mu \mathrm{M}, 100 \mu \mathrm{M})$, as indicated. Quantitative realtime RT-PCR was performed as described in Materials and Methods. mRNA levels were compared to known standard samples and were normalized to GAPDH. Results are mean values (+/-SEM, $n=5)$. One-way ANOVA demonstrated a significant difference $(p<0.05)$ between the control and the treatment; (b) Analysis of Oct4 mRNA expression in HDFa cells following Oct4 lentivirus transfection. HDFa were transfected with lentivirus and isolated RNA was subjected to quantitative RT-PCR. mRNA levels were compared to known standard samples and normalized to GAPDH. Results are mean values (+/-SEM, $n=3)$; $(\mathrm{c})$ Expression of Oct4 mRNA in HDfa cells subjected to Oct4-expressing lentivirus infection and HDAC or DNMT inhibition. HDFa cells were transfected with or without Oct4 lentivirus and treated for 7 days with VPA $(5 \mathrm{mM})$ or the DNMT inhibitor, 5 -azacytidine $(5$-aza, $5 \mu \mathrm{M}))$ as indicated prior to RNA isolation. Total RNA was isolated and subjected to quantitative RT-PCR. Expression levels were compared to known standard samples and normalized to GAPDH. Results are mean values (+/-SEM, $n=3)$. mRNA levels for Control, VPA and 5-aza samples are also presented in numerical form for comparison to lentivirus-infected samples. One-way ANOVA demonstrated a significant difference $(p<0.05)$ between the control and treatments; (d) Analysis of Nanog mRNA expression in HDFa cells following Nanog lentivirus transfection. HDFa were transfected with lentivirus expressing Nanog and isolated RNA was subjected to quantitative RT-PCR. mRNA levels were compared to known standard samples and normalized to GAPDH. Results are mean values (+/-SEM, $n=3$ ). mRNA levels for Control, VPA and 5-aza samples are also presented in numerical form for comparison to lentivirus-infected samples. One-way ANOVA demonstrated a significant difference $(p<0.05)$ between the control and the TSA colonies.

were observed and the number of colonies in each well was determined. TSA pre-treatment resulted in a more than 2-fold increase in colony formation relative to untreated control cultures (Figure 3(c)). Similar results were observed when comparing Scriptaid- and VPA-pretreated cultures with their respective un-treated control cultures (data not shown).

AdiPS-Ctl and AdiPS-TSA cell lines were obtained by clonal expansion of the control and TSA colonies, re- spectively (Figure 3(b), right panel). Cells were characterized with respect to alkaline phosphatase staining, a marker for early ES cell formation, Oct4/TRA1-60 (red/ green) and Sox2/TRA1-60 (red/green) combination staining and by the formation of embryoid bodies (EB) (Figure 3(d)). TRA1-60 is a surface marker for matured iPS cells [29] and embryoid bodies represent the earliest stages of differentiation and embryo development in vitro. ES cell-like colonies from pre-treated cultures were in- 


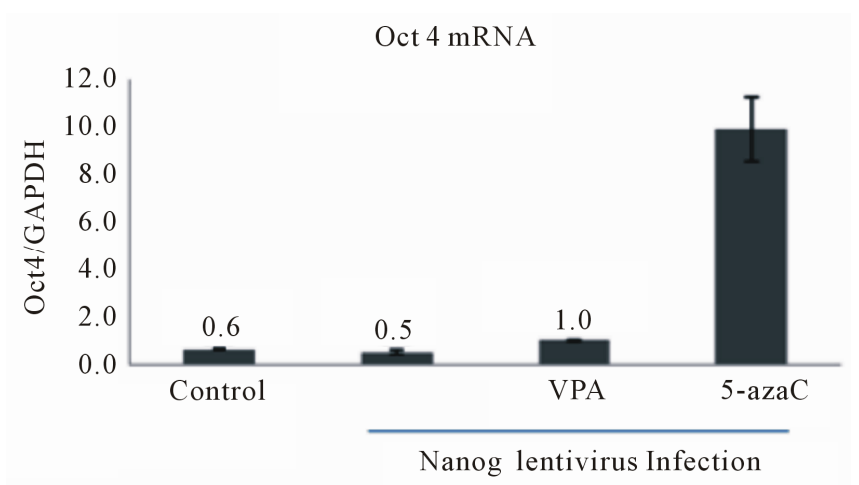

(a)

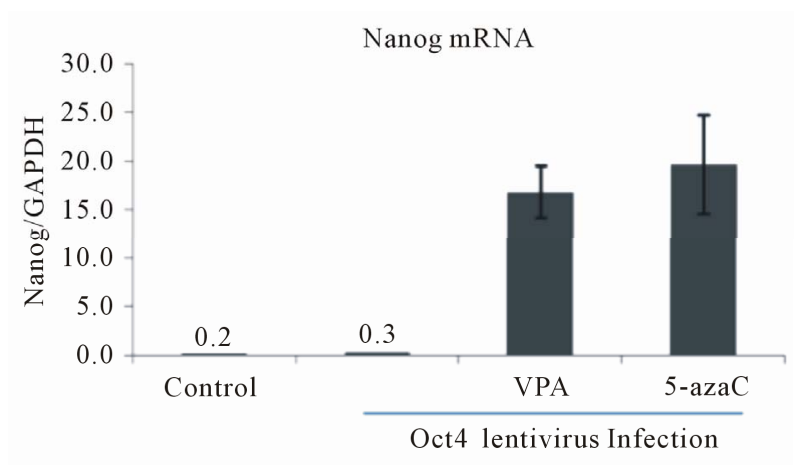

(b)

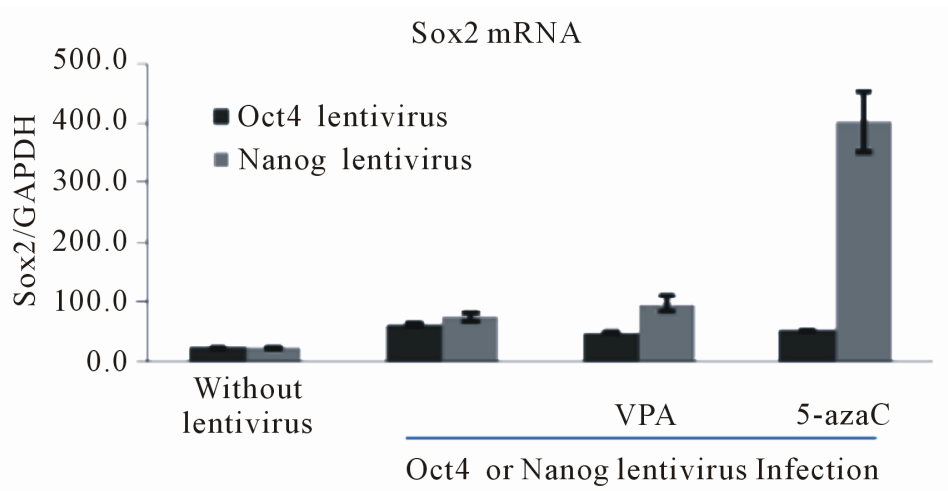

(c)

Figure 2. Reciprocal activation of endogenous pluripotency factor genes in Oct4 or Nanog lentivirus-infected HDF cells by treatment with HDAC or DNMT inhibitors. (a) Expression of Oct4 mRNA in HDFa cells subjected to Nanog-expressing lentivirus infection and HDAC or DNMT inhibition. HDFa cells were transfected with or without Nanog lentivirus and treated with VPA or 5-azacytidine as indicated. Total RNA was isolated and subjected to quantitative RT-PCR. Expression levels were compared to known standard samples and normalized to GAPDH. Results are mean values (+/-SEM, $n=3)$. One-way ANOVA demonstrated a significant difference $(p<0.05)$ between the control and the treatment; (b) Expression of Nanog mRNA in HDFa cells subjected to Oct4-expressing lentivirus infection and HDAC or DNMT inhibition. HDFa cells were transfected with or without Oct4 lentivirus and treated with VPA or 5-azacytidine as indicated. Results are mean values (+/-SEM, $n=3$ ). One-way ANOVA demonstrated a significant difference $(p<0.05)$ between the control and the treatment; (c) Expression of Sox 2 mRNA in HDFa cells subjected to Oct4- or Nanog-expressing lentivirus infection and HDAC or DNMT inhibition. HDFa cells were transfected with or without Oct4 lentivirus and treated with VPA or 5-azacytidine as indicated. Dark bars indicate Sox2 mRNA levels in Oct4 lentivirus infected cells and the light bars Sox2 levels in Nanog-expressing lentivirus-infected cells.

distinguishable from control colonies with respect to the parameters analyzed. Thus, HDACi treatment increases the formation of colonies that are grossly similar to control colonies.

Realtime RT-PCR also revealed a $10^{3}-10^{4}$-fold increase in Oct4 and Nanog mRNA levels between preadipocyte and AdiPS-Ctl or AdiPS-TSA cells (Figure 3(e), top panel) consistent with the expected changes associated with cell reprogramming. In addition, bisulfite sequencing demonstrated extensive demethylation (open circles) of the promoter regions of Oct4 and Nanog genes in AdiPS-Ctl or AdiPS-TSA cells relative to preadipocyte colonies (Figure 3(e), bottom panel).

We next compared pluripotency marker gene expression levels in AdiPS cells with the levels in preadipocyte and hES cells (Mel-1) by low density microarray. As expected, both AdiPS-Ctl and AdiPS-TSA cells showed similar gene expression patterns to Mel-1 hES cells and strikingly different profiles than preadipocyte cells (Figure 3(f)). Expression of ectopic pluripotency genes was not detected in established AdiPS cell lines (data not shown). These data suggest that AdiPS clonal cell lines more closely resemble hES cells than their preadipocyte progenitors with respect to pluripotency gene expression.

To determine if AdiPS cells display functional similarities to hES cells, we next conducted in vitro and in vivo differentiation experiments. Human AdiPS cells were first transferred to Matrigel plates and treated with high-serum, low growth factor medium for 3 to 4 weeks. Live-image microscopy revealed beating cardiomyocytes (white arrows) (Figure 4(a)). AdiPS cells were also injected into the interscapular region of athymic Nude mice 
Human preadipocytes

$\sim 10^{5}$ cells/well

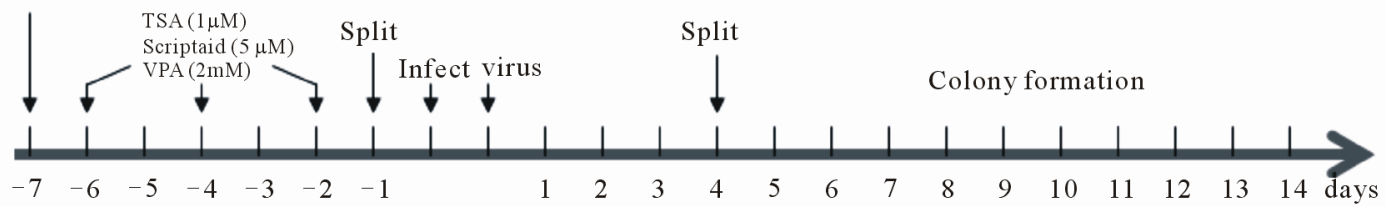

Fibroblast culture $+\mathrm{HDACi}$

(a)
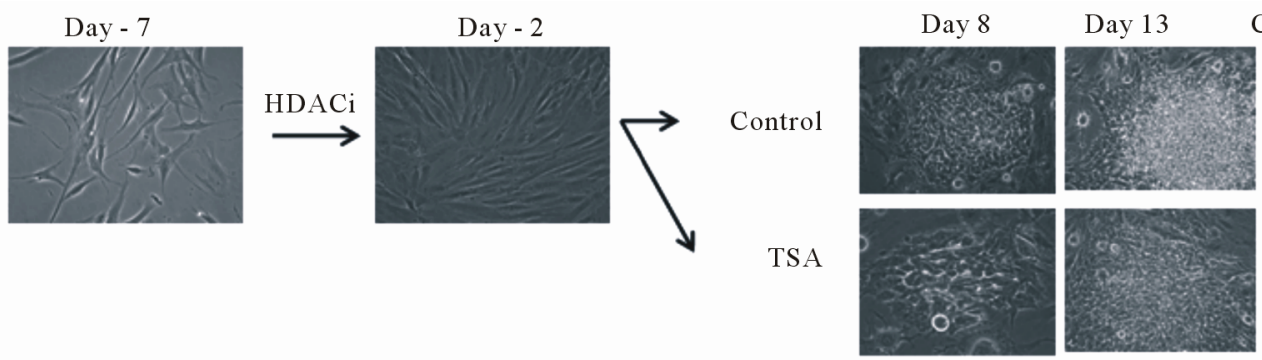

Clonal Expansion

(b)

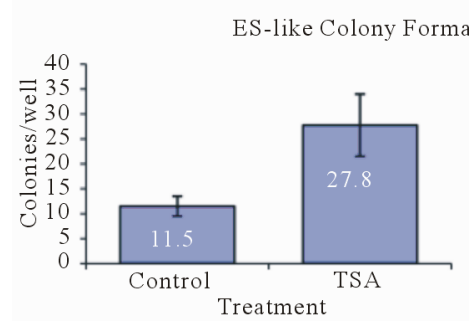

(c)

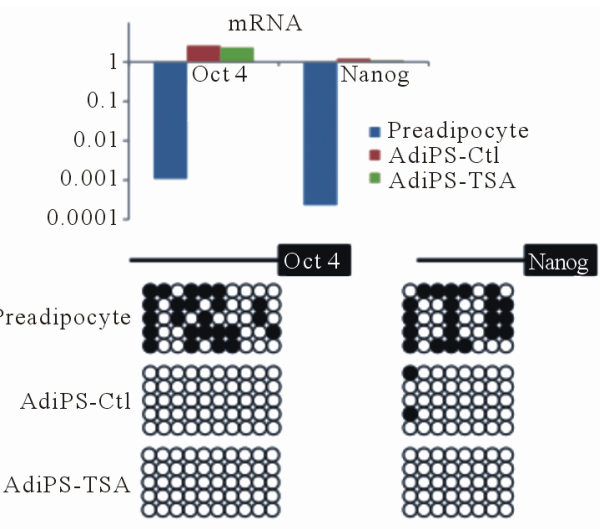

(e)

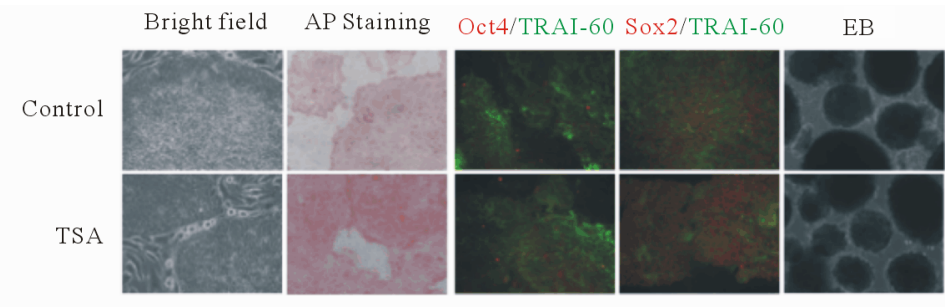

(d)

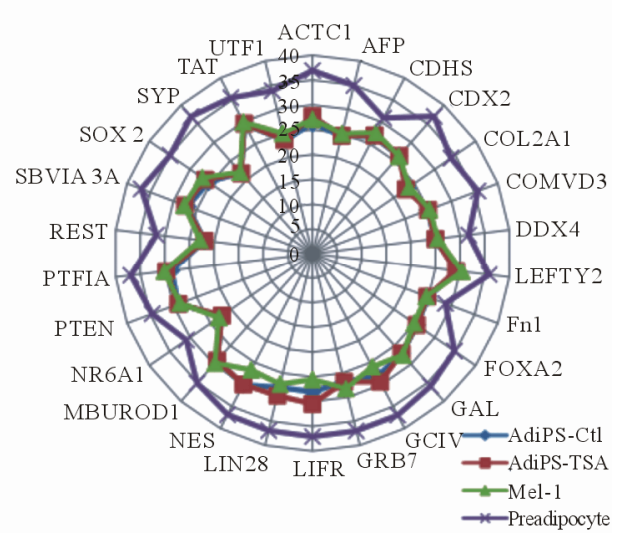

(f)

Figure 3. Characterization of adipose induced pluripotent stem (AdiPS) cells produced by pre-treatment of preadipocytes with epigenetic modulators. (a) Experimental strategy for production of AdiPS cells; (b) Left Panel: Preadipocytes treated with HDAC inhibitors (HDACi). Images show and increase in cell density and changes in cell morphology during treatment. Right Panel: ES cell-like colonies from control and TSA-treated cultures showing well-defined boundaries; (c) The mean number of ES cell-like colonies formed in TSA-treated and untreated control cultures; (d) Analysis of morphology and immunostaining for the pluripotency marker (Oct4, Sox2) and matured iPS/ES cells (TRA1-60) in control and TSA-treated AdiPS cells. Embryoid bodies (EB). Alkaline phosphatase (AP); (e) Top panel: Oct4 and Nanog mRNA expression levels in preadipocytes and in control adipose-derived iPS cell (AdiPS-Ctl) and TSA-treated adipose-derived iPS cell (AdiPS-TSA) cultures. Bottom panel: DNA methylation status of Oct4 and Nanog promoter regions from human preadipocyte, AdiPS-Ctl and AdiPS-TSA cells. Open circles: unmethylated CpG dinucleotides; closed circles; methylated CpG dinucleotides; (f) Low density microarray analysis comparing pluripotency gene expression in preadipocyte, hES cell (Mel-1), AdiPS-Ctl and AdiPS-TSA cells. Values indicate relative quantitation by the $\Delta \Delta \mathrm{C}_{\mathrm{T}}$ method. 

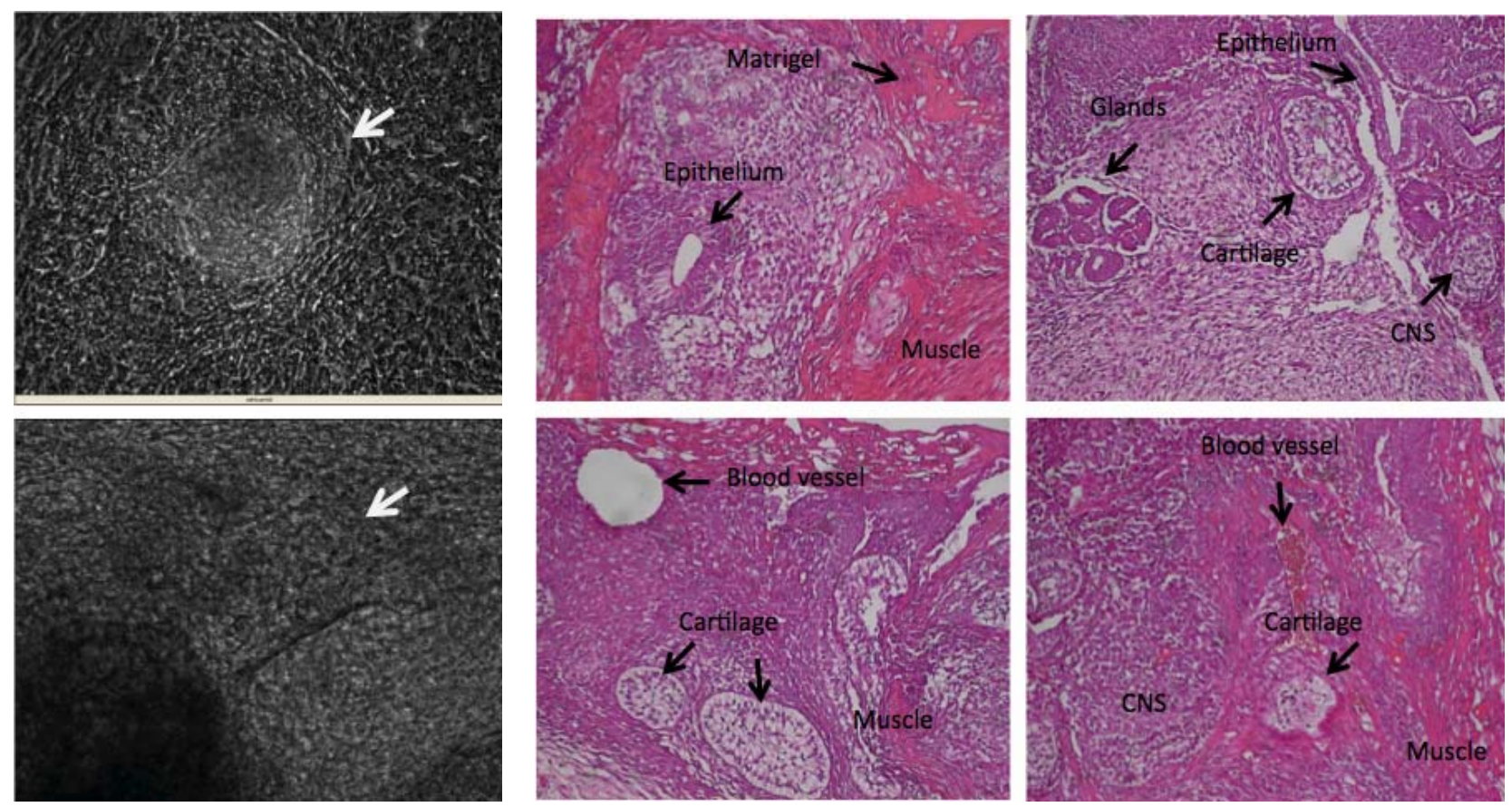

(a)

(b)

Figure 4. Differentiation of AdiPS cells in vitro and in vivo. (a) Morphology of beating cardiomyocytes. Human AdiPS cells were transferred onto a Matrigel plate and treated with high serum/low growth factor medium for 3 to 4 weeks. Live images of in vitro-generated beating cardiomyocytes (white arrows) were captured by microscopy; (b) Morphology of formed teratomas. Human AdiPS cells were injected into athymic nude mice and after 6 to 7 weeks, samples were collected and stained with hemotoxylin-eosin (H\&E). These four panels represent different sections through a teratoma illustrating tissues of all three embryonic germ layers as indicated; epithelium, muscle, cartilage, glands, central nervous system (CNS).

to assess their capacity for teratoma formation. Teratoma masses were observed 6 - 7 weeks after injection and H\&E staining demonstrated tissues representing all three germ layers (Figure 4(b)). Comparison of preadipocytes with human adipose-derived adult stem cells, isolated from subcutaneous fat, was indistinguishable with respect to their responses to HDAC inhibitor pre-treatment (data not shown).

The expression status of the Dlk1-Dio3 imprinted gene cluster reflects the potential for production of high-grade chimaeras and their viability [30]. Interestingly, HDAC inhibitor treatment reactivates Dlk1-Dio3 expression within partially reprogrammed cells resulting in the establishment of fully reprogrammed mouse iPS cells. High levels of Dlk1 (also known as Pref1) mRNA have also been detected in mouse preadipocytes and these levels dramatically decrease during adipocyte differentiation [31]. Therefore, we sought to determine the effect of the HDAC inhibitor, TSA, on the Dlk1 gene in our human AdiPS cells. Both types of AdiPS cells and hES cells were found to express high levels of Dlk1 mRNA as compared to human preadipocytes (Figure 5(a)). However, AdiPS-TSA cells had higher levels of Dlk1 gene expression than AdiPS-Ctl cells. The promoter region of the imprinted Dlk1 gene harbors hemimethylated $\mathrm{CpG}$ dinucleotides [32]. This region also contains a CTCF insulator binding motif (CCGCnnGGnGGg/tC) as indicated by the box in Figure 5(b). Analysis of this region using bisulfite sequencing revealed differential hemimethylation of $\mathrm{CpG}$ dinucleotides between preadipocytes, hES cells and our AdiPS cells in the region of the CTCF binding site (Figures 5(c) and (d)). Specifically, human preadipocytes showed higher levels of hemi-methylation than Mel-1, AdiPS-Ctl and AdiPS cells (Figure 5(d)). These results agree well with published reports [30] and suggest that pretreatment of human preadipocytes with a broad range HDAC inhibitor such as TSA, might improve the quality of reprogrammed cells.

\subsection{Induction of ES Cell-Like Colony Maturation by Post-Treatment of Cells with an HDAC Inhibitor}

It has been shown that only a small percentage of ES cell-like colonies become fully reprogrammed and acquire hES cell characteristics such as pluripotency and self-renewal. Thus, colony formation alone is necessary, but not sufficient, for pluripotentcy of ES-like cells. The acquisition of pluripotency and self-renewal has been termed colony maturation and we next sought to determine how post-treatment of lentivirus-transfected cells 


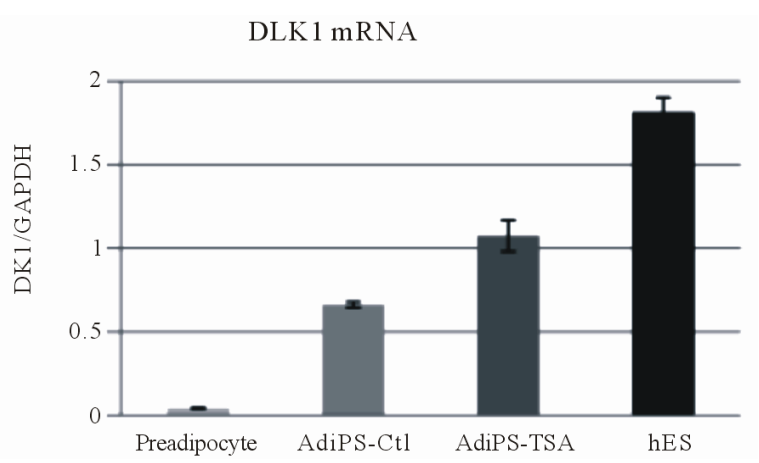

(a)
DLK CpG island \#1

GTCCTCTTGCTCCTGCTGGCTTTCGGCCACAGCACCTATGGTGAGTTCC CCGGCGGCCCGGCTCGCGCCCCCTCTGGGGAAGCCTGCGACTCCCCGCC GGCCGCCCGTTCOCCGCACGCCCCGTCTCGTGAGCCCCAACTCCGCC

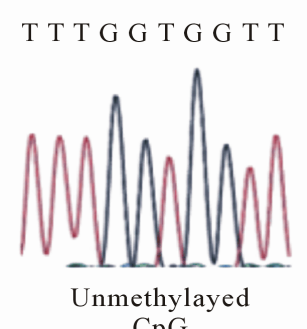

$\mathrm{CpG}$

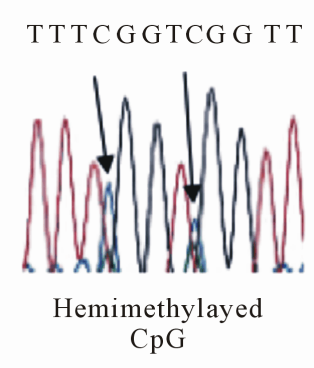

(c) (b)

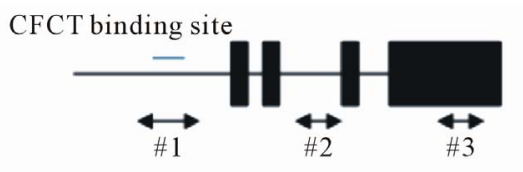

Preadipocyte
Mel-1
AdiPS-Ctl
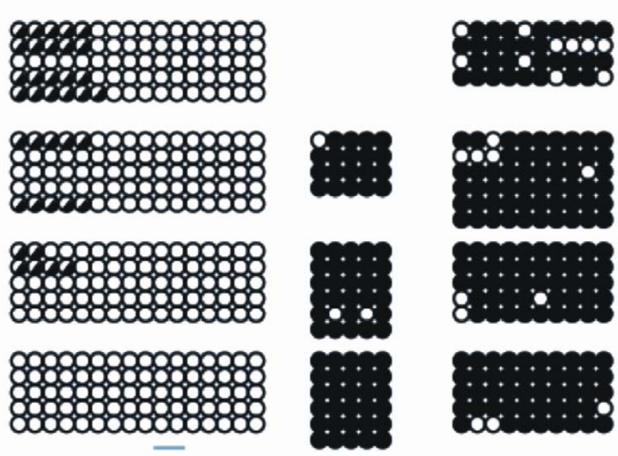

CTCF binding site

OUnmethylayed $\mathrm{CpG}$

- Methylated $\mathrm{CpG}$

- Hemimethylayed $\mathrm{CpG}$

(d)

Figure 5. Dlk1 mRNA expression and DNA methylation of the Dlk1 promoter. (a) Dlk1 mRNA levels from human preadipocytes, hES cells and AdiPS cells (AdiPS-Ctl, AdiPS-TSA) were determined by Real-time RT-PCR and expression was normalized to GAPDH; (b) Nucleotide sequence of the Dlk1 promoter. CpG dinucleotides are underlined and the putative CTCF binding site is shown within the box; (c) Representative chromatograms of unmethylated and hemimethylated CpG dinuleotides within the Dlk1 gene promoter; (d) DNA methylation status of the human Dlk1 gene. Analysis of three genomic regions (\#1 - \#3) by bisulfite sequencing. Black boxes and horizontal line denote exons and introns, respectively. Empty circles, unmethylated CpG dinucleotides; filled circles, methylated $\mathrm{CpG}$ dinucleotides; half-filled circles, hemimethylated $\mathrm{CpG}$ dinucleotides.

with the epigenetic modifier, zebularine, affects this process. Our experimental strategy is outlined in Figure 6(a). TSA $(1 \mu \mathrm{M})$ or zebularine $(50 \mu \mathrm{M})$ pre-treated human preadipocytes were transfected with lentivirus as previously described (see Figure 3(a)). One day after transferring to a mouse embryonic fibroblast feeder layer, hES culture medium was supplemented with zebularine as indicated. Colonies were first visible 3 days later (Figure 6(b)). These colonies were tracked through clonal expansion and cell lines were established (Figure 6(b)) as described in our earlier experiment (see Figure 3(a)). Tightly packed colonies growing on the feeder layer were stained with alkaline phosphatase (AP), and double immunostained for Sox2/TRA1-60 (red/green) (Figure 6(c)), markers for colony formation and maturation, respectively. The percentage of positive colonies in each treatment group is shown in Figure 6(d). All treatment regimens produced colonies that were positive for AP. However, only $50 \%-60 \%$ of the colonies from cells pretreated with TSA or Zeb stained positive for Sox2. More colonies from cells pretreated with zebularine (Zeb) showed TRA1-60 staining $(\sim 22 \%)$ than control $(\mathrm{Ctl})$ colonies $(8 \%)$ or colonies from cells pretreated with TSA $(8 \%)$. Moreover, zebularine post-treatment increased the 
Human preadipocytes

$10^{5}$ cells/well

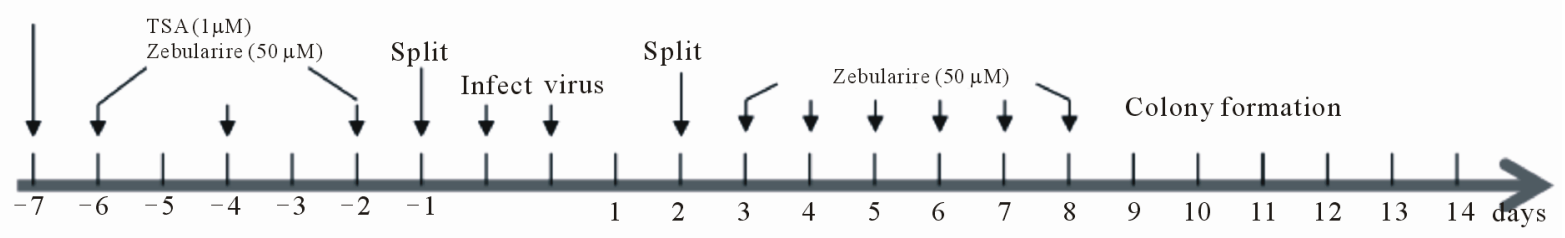

HDACi/DNMTi

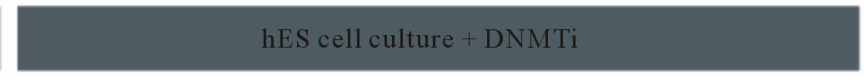

(a)

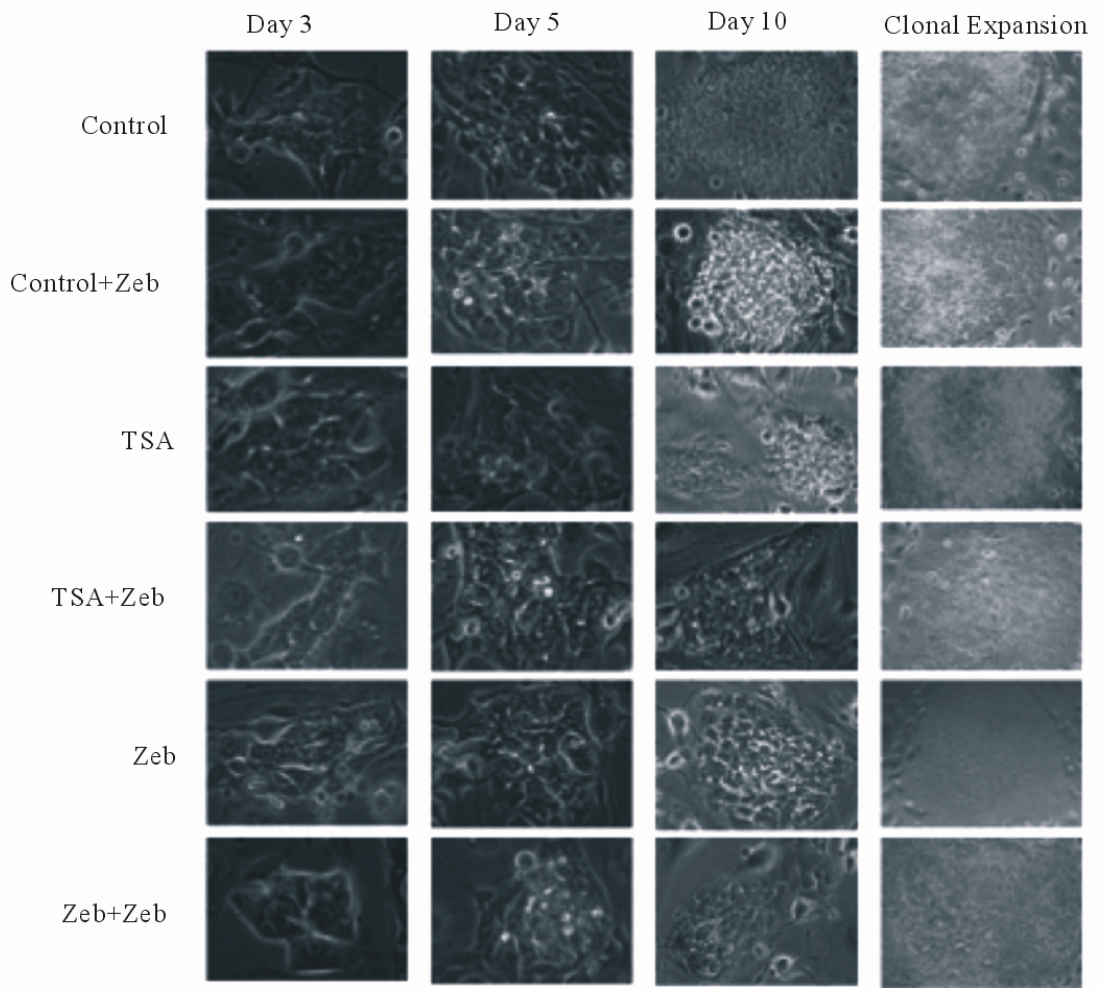

(b)
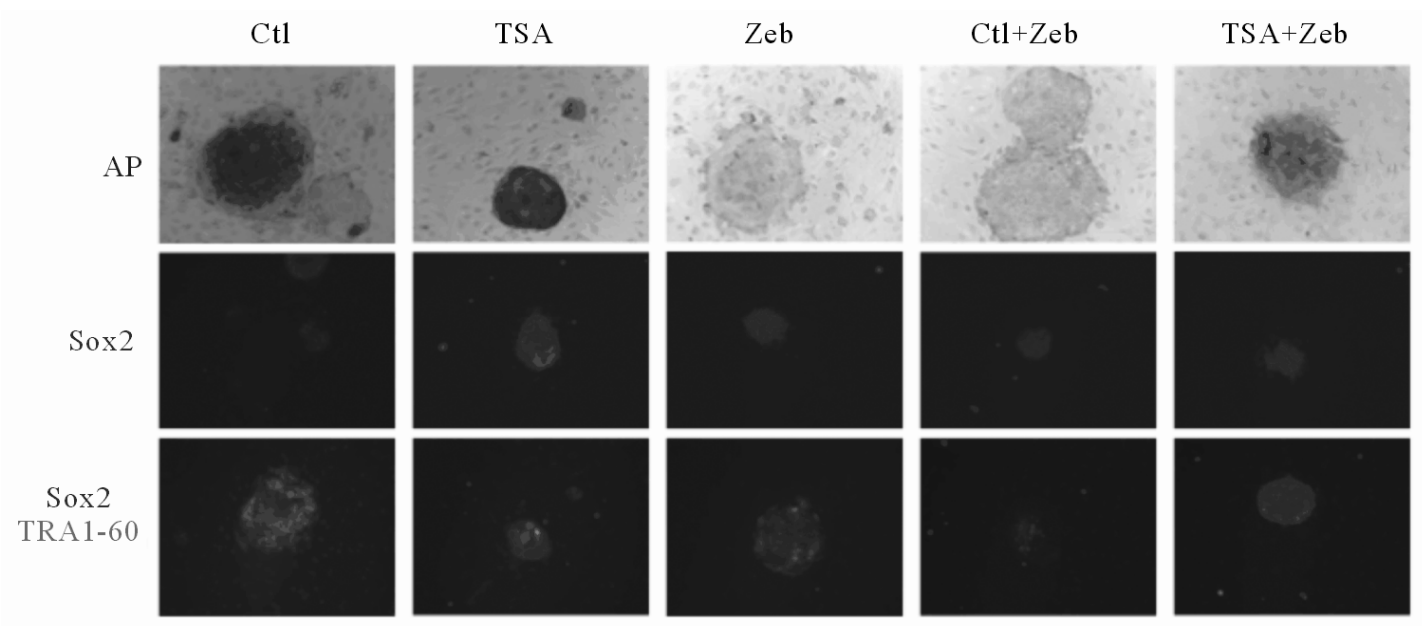

$\mathrm{Zeb}+\mathrm{Zeb}$
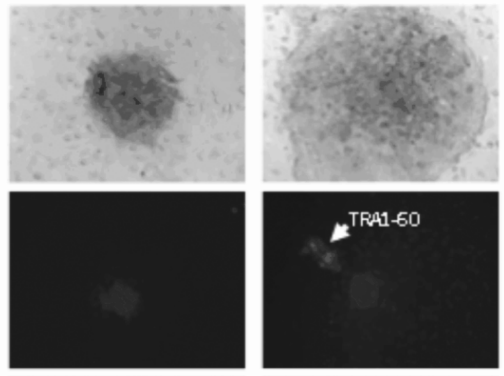

(c) 


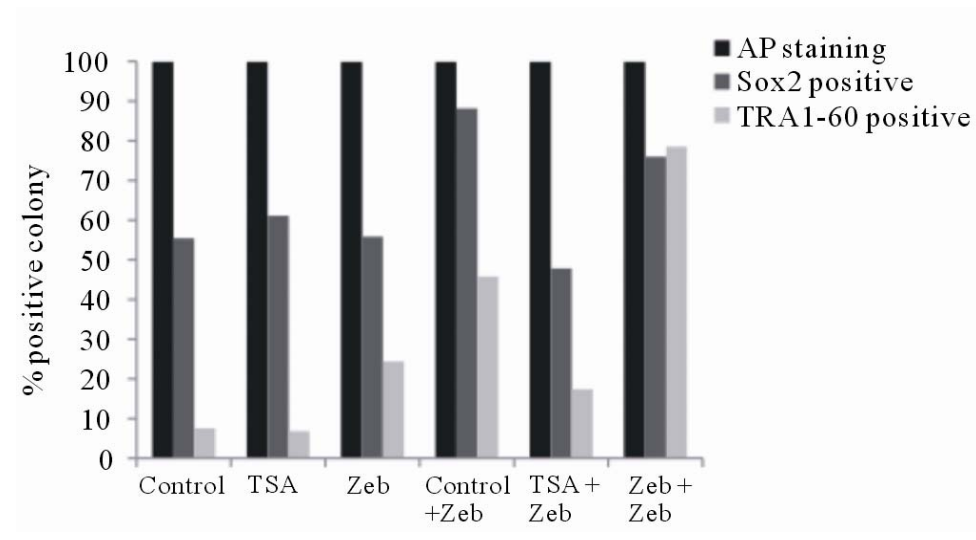

(d)
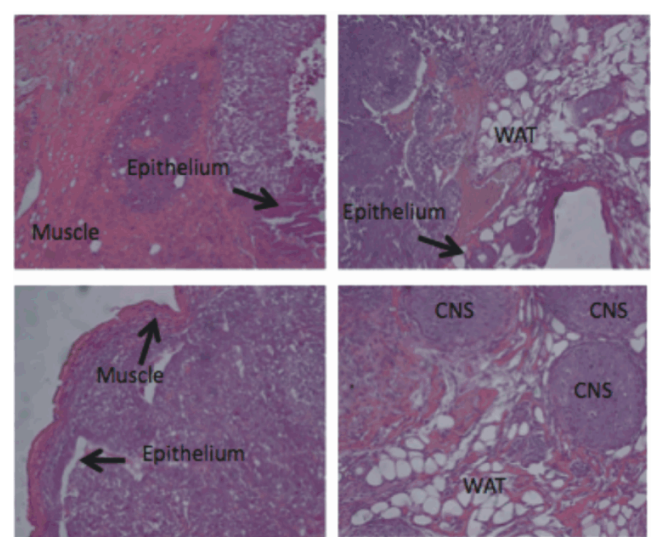

(e)

Figure 6. Characterization of adipose induced pluripotent stem (AdiPS) cells produced by pre- and post-treatment of preadipocytes with epigenetic modulators. (a) Experimental scheme for AdiPS cell production; (b) Morphological progression of ES cell-like colony formation in preadipocytes treated with the epigenetic modulators, TSA $(1 \mu \mathrm{M})$ and/or zebularine $(50 \mu \mathrm{M})$; (c) Examples of ES cell-like colonies stained for alkaline phosphatase (AP), Sox2 and Sox2/TRA1-60; (d) The percentage of positive-staining colonies observed in each experimental group is shown in Figure 6(c); (e) Teratoma formation in nude mice following AdiPS cell injection. All three embryonic germ layers are visible by hematoxylin-eosin (H\&E) staining; epithelium, muscle, white adipocytes (WAT), central nervous system (CNS).

number of both Sox2 (88\%) and TRA1-60 (42\%) expressing colonies as compared to colonies from cells pretreated with TSA $(50 \%-60 \%$ Sox 2 and $8 \%$ TRA $1-60)$ or zebualrine $(50 \%-60 \%$ Sox 2 and $22 \%$ TRA $1-60)$. Somewhat surprisingly, colonies from cells pretreated with TSA and subsequently post treated with zebularine had reduced Sox $2(\sim 42 \%)$ and TRA1-60 (18\%) expression compared to colonies from cells only post-treated with zebularine. In contrast, pretreatment and post-treatment of cells with zebularine resulted in greater than $75 \%$ of the colonies staining positive for Sox 2 and TRA1-60. Realtime RT-PCR of established AdiPS cell lines confirmed that Sox 2 (or Oct4 or Nanog) immunostaining was not due to ectopic gene expression (data not shown). Established AdiPS cells were also injected into Nude mice to assess their potential for teratoma formation. All three germ layers were present in the recovered tissue masses indicating teratoma formation (Figure 6(e)).

\section{DISCUSION}

We have generated human adipose tissue-derived iPS (AdiPS) cells from primary preadipocytes and adiposederived adult stem cells using epigenetic modulation combined with lentivirus-induced pluripotency factor expression. Our results demonstrate three important points. First, treatment of cells with HDAC or DNMT inhibitors, either before or after lentivirus transfection, increases pluripotency factor expression. These data sug- gest that epigenetic modification may activate both the autotranscriptional and the reciprocal transcriptional regulation pathways of the endogenous pluripotency factors, Oct4 and Nanog, in adult human cells. Second, epige- netic modulation of adipose tissue-derived cells, before lentivirus-induced pluripotency factor expression, increases the efficiency of ES cell-like colony formation. Third, DNMT inhibition, both before and after ES celllike colony formation, increases the efficiency of colony maturation. Taken together, these data raise the interesting possibility that selective, small molecule-based epigenetic modulation may promote pluripotency transcription factor expression and in turn, provide a more efficient means for iPS cell production.

Adipose tissue is likely to be an excellent source for cell reprogramming for at least two reasons. First, adipose tissue, like bone marrow, maintains adult mesenchymal stem cells that can differentiate toward the osteogenic, adipogenic, neurogenic, myogenic, and chondrogenic lineage [33-35]. Second, it has been demonstrated that adipose derived stem cells express high levels of pluripotent transcription factors such as Oct4, Sox2, Rex1, Klf4, cMyc, and Esrr $\beta$ [28,35]. Therefore, these cells may provide a more favorable template for reprogramming to ES-like cells due to their greater plasticity for reprogramming coupled with their broader differentiation potential relative to non-adipose-derived tissue sources. Moreover, adipose cells may also provide an avenue for creating model "disease-in-a-dish" systems that exploit natural variations among fat depots associated with divergent metabolic responses.

Numerous groups are attempting to bypass viral vector-mediated reprogramming factor over-expression as a cell reprogramming strategy. Recent alternative strategies include targeting the activation of self-renewal while inhibiting differentiation by controlling cell-signaling 
pathways. Approaches include inhibitors of MEK [36], GSK [37], ROCK [36], and TGF $\beta$ [38]. In addition, p53 inhibition has been reported to improve reprogramming [39] as well as the application of a $\mathrm{Ca}^{2+}$ channel activator [40]. Inhibitors of epigenetic modifications that target DNMT [12,40], HDAC [12], lysine methylase [37], and HMT [40] enable more efficient viral-based reprogramming through single-factor (Oct4) or dual-factor (Oct4 and Sox2) over-expression [38,41]. Recently, Yuan and colleagues reported that the protein, arginine methylatransferase inhibitor (AMI-5), in combination with a TGF $\beta$ inhibitor, enabled Oct4 single-factor induced reprogramming of mouse embryonic fibroblasts [24]. These and other results indicate that a pure chemical-based reprogramming strategy for somatic cells is indeed, feasible.

\section{CONCLUSION}

In this study, we identified multiple effects of small molecule epigenetic modulators on somatic cell reprogramming. Primarily, we found that HDAC inhibition, prior to reprogramming factor transfection, increases ES cell-like colony formation up to 3-fold as compared to non-treated controls. Furthermore, DNMT inhibition before and after reprogramming factor induction promotes maturation of partially reprogrammed cells and yields $\sim 4$-fold more mature colonies than the control group. Thus, our data demonstrate that small molecule-based epigenetic modulation can be used to augment existing virus-based reprogramming regimens. In addition, our work here may also provide valuable information regarding the development of virus-independent, small molecule-based reprogramming strategies that target restrictive epigenetic modifications that are established during differentiation.

\section{ACKNOWLEDGEMENTS}

The authors thank Dr. David Burk and Ms. Susan Newman for technical advice. This work was funded by the Louisiana Board of Regents Industrial Ties Program Grant LEQSF (2008-11)-RD-B-06 and by NIH Small Business Innovation Research grant (1 R43 HL104977). We also acknowledge the use of Genomics, Cell Biology and Imaging Core facilities that are supported, in part, by COBRE (NIH P20- RR021945) and NORC (NIH 1P30-DK072476) center grants from the NIH.

\section{REFERENCES}

[1] Takahashi, K. and Yamanaka, S. (2006) Induction of pluripotent stem cells from mouse embryonic and adult fibroblast cultures by defined factors. Cell, 126, 663-676. doi:10.1016/j.cell.2006.07.024

[2] Park, I.-H., Arora., N., Huo, H.G., Maherali, N., Ahfeldt, T., Shimamura, A., Lensch, M.W., Cowan, C., Hochedlin- ger, K. and Daley, G. Q. (2008) Disease-Specific induced pluripotent stem cells. Cell, 134, 1-10. doi:10.1016/j.cell.2008.07.041

[3] Yu, J.Y., Vodyanik, M.A., Smuga-Otto, K., AntosiewiczBourget, J., Frane, J.L., Tian, S., Nie, J., Jonsdottir, G.A., Ruotti, V., Stewart, R., Slukvin, I.I. and Thomson, J.A. (2007) Induced pluripotent stem cell lines derived from human somatic cells. Science, 318, 1917-1920. doi:10.1126/science. 1151526

[4] Meng, X.L., Shen, J.S., Kawagoe, S., Ohashi, T., Brady, R.O., et al. (2010) Induced pluripotent stem cells derived from mouse models of lysosomal storage disorders. Proceedings of the National Academy of Sciences of the USA, 107, 7886-7891. doi:10.1073/pnas.1002758107

[5] Ebert, A.D., Yu, J.Y., Rose, F.F. Jr., Mattis, V.B., Lorson, C.L., et al. (2009) Induced pluripotent stem cells from a spinal muscular atrophy patient. Nature, 457, 277-280.

[6] Lee, G., Papapetrou, E.P., Kim, H., Chambers, S.M., Tomishima, M.J., et al. (2009) Modelling pathogenesis and treatment of familial dysautonomia using patientspecific iPSCs. Nature, 461, 402-406.

[7] Rashid, S.T., Corbineau, S., Hannan, N., Marciniak, S.J., Miranda, E., et al. (2010) Modeling inherited metabolic disorders of the liver using human induced pluripotent stem cells. The Journal of Clinical Investigation, 120, 3127-3136. doi:10.1172/JCI43122

[8] Zhang, N., An, M.C., Montoro, D., Ellerby, L.M. (2010) Characterization of human huntington's disease cell model from induced pluripotent stem cells. PLoS Currents, 2, RRN1193. doi:10.1371/currents.RRN1193

[9] Zhao, T. and Xu, Y. (2010) p53 and stem cells: New developments and new concerns. Trends in Cell Biology, 20, 170-175. doi:10.1016/j.tcb.2009.12.004

[10] Yu, J.Y., Hu, K.J., Smuga-Otto, K., Tian, S.L., Stewart, R., Slukvin, I.I. and Thomson, J.A., (2009) Human induced pluripotent stem cells free of vector and transgene sequences. Science, 10, 797-801. doi:10.1126/science. 1172482

[11] Sommer, C.A., Stadtfeld, M., Murphy, G.J., Hochedlinger, K., Kotton, D.N., et al. (2009) Induced pluripotent stem cell generation using a single lentiviral stem cell cassette. Stem Cells, 27, 543-549.

doi:10.1634/stemcells.2008-1075

[12] Huangfu, D.W., Maehr, R., Guo, W.J., Eijkelenboom, A., Snitow, M., Chen, A.E. and Melton, D.A. (2008) Induction of pluripotent stem cells by defined factors is greatly improved by small-molecule compounds. Nature Biotechnology, 26, 795-797.

[13] Okita, K., Nakagawa, M., H., Hong, Ichisaka, T. and Yamanaka, S., (2008) Generation of mouse induced pluripotent stem cells without viral vectors. Science, 322, 949953. doi: $10.1126 /$ science. 1164270

[14] Warren, L., Manos, P.D., Ahfeldt, T., Loh, Y.-H., Li, H., et al. (2010) Highly efficient reprogramming to pluripotency and directed differentiation of human cells with synthetic modified mRNA. Cell Stem Cell, 7, 618-630. doi:10.1016/i.stem.2010.08.012

[15] Yakubov, E., Rechavi, G., Rozenblatt, S. and Givol, D. 
(2010) Reprogramming of human fibroblasts to pluripotent stem cells using mRNA of four transcription factors. Biochemical and Biophysical Research Communications, 394, 189-193. doi:10.1016/j.bbrc.2010.02.150

[16] Anokye-Danso, F., Trivedi, C.M., Juhr, D., Gupta, M., Cui, Z., et al. (2011) Highly efficient miRNA-mediated reprogramming of mouse and human somatic cells to pluripotency. Cell Stem Cell, 8, 376-388. doi:10.1016/j.stem.2011.03.001

[17] Woltjen, K., Michael, I.P., Mohseni, P., Desai, R., Mileikovsky, M., et al. (2009) piggyBac transposition reprograms fibroblasts to induced pluripotent stem cells. Nature, $\mathbf{4 5 8}$, 766-770.

[18] Kaji, K., Norrby, K., Paca, A., Mileikovsky, M., Mohseni, P., et al. (2009) Virus-Free induction of pluripotency and subsequent excision of reprogramming factors. Nature, 458, 771-775.

[19] Kim, D., Kim, C.-H, Moon, J.-I., Chung, Y.-G., Chang, M.-Y., Han, B.-S., Ko, S., Yang, E., Cha, K.Y., Lanza, R. and Kim, K.-S. (2009) Generation of human induced pluripotent stem cells by direct delivery of reprogramming proteins. Cell Stem Cell, 4, 472-476. doi:10.1016/j.stem.2009.05.005

[20] Zhou, H.Y., Wu, S.L., Joo, J.Y., Zhu, S.Y., Han, D.W., Lin, T.X., Trauger, S., Bien, G., Yao, S., Zhu, Y., Siuzdak, G., Scholer, H.R., Duan, L.X. and Ding, S. (2009) Generation of induced pluripotent stem cells using recombinant proteins. Cell Stem Cell, 4, 381-384.

[21] Zhu, S.Y., Li, W.L., Zhou, H.Y., Wei, W.G., Ambasudhan, R., et al. (2010) Reprogramming of human primary somatic cells by OCT4 and chemical compounds. Cell Stem Cell, 7, 651-655. doi:10.1016/j.stem.2010.11.015

[22] Li, Y., Zhang, Q., Yin, X., Yang, W., Du, Y., et al. (2011) Generation of iPSCs from mouse fibroblasts with a single gene, Oct4, and small molecules. Cell Research, 21, 196204. doi: $10.1038 / \mathrm{cr} .2010 .142$

[23] Chen, J.K., Liu, J., Yang, J.Q., Chen, Y., Chen, J., et al. (2011) BMPs functionally replace Klf4 and support efficient reprogramming of mouse fibroblasts by Oct4 alone. Cell Research, 21, 205-212. doi:10.1038/cr.2010.172

[24] Yuan, X., Wan, H., Zhao, X., Zhu, S., Zhou, Q., et al. (2011) Combined chemical treatment enables Oct4-induced reprogramming from mouse embryonic fibroblasts. Stem Cells, 29, 549-553. doi:10.1002/stem.594

[25] Zhu, S., Wei, W. and Ding, S. (2011) Chemical strategies for stem cell biology and regenerative medicine. Annual Review of Biomedical Engineering, 13, 73-90.

[26] Jaenisch, R. and Young, R. (2008) Stem cells, the molecular circuitry of pluripotency and nuclear reprogramming. Cell, 132, 567-582. doi:10.1016/j.cell.2008.01.015

[27] Kim, J., Chu, J.L., Shen, X.H., Wang, J.L. and Orkin, S.H. (2008) An extended transcriptional network for pluripotency of embryonic stem cells. Cell, 132, 1049-1061. doi:10.1016/j.cell.2008.02.039

[28] Sun, N., Panetta, N.J., Gupta, D.M., Wilson, K.D., Lee, A., et al. (2009) Feeder-Free derivation of induced pluripotent stem cells from adult human adipose stem cells. Proceedings of the National Academy of Sciences of the
USA, 106, 15720-15725. doi:10.1073/pnas.0908450106

[29] Chan, E.M., Ratanasirintrawoot, S., Park, I.-H., Manos, P.D., Loh, Y.-H., et al. (2009) Live cell imaging distinguishes bona fide human iPS cells from partially reprogrammed cells. Nature Biotechnology, 27, 1033-1037.

[30] Stadtfeld, M., Apostolou, E., Akutsu, H., Fukuda, A., Follett, P., et al. (2010) Aberrant silencing of imprinted genes on chromosome $12 \mathrm{qF} 1$ in mouse induced pluripotent stem cells. Nature, 465, 175-181.

[31] Sul, H.S., Smas, C., Mei, B. and Zhou, L. (2000) Function of pref-1 as an inhibitor of adipocyte differentiation. International Journal of Obesity and Related Metabolic Disorders, 24, 15-19.

[32] Wylie, A.A., Murphy, S.K., Orton, T.C. and Jirtle, R.L. (2000) Novel imprinted DLK1/GTL2 domain on human chromosome 14 contains motifs that mimic those implicated in IGF2/H19 regulation. Genome Research, 10, 1711-1718. doi:10.1101/gr.161600

[33] Zuk, P.A., Zhu, M., Ashjian, P., De Ugarte, D.A., Huang J.I., et al. (2002) Human adipose tissue is a source of multipotent stem cells. Molecular Biology of the Cell, 13, 4279-4295. doi:10.1091/mbc.E02-02-0105

[34] Zuk, P.A., Zhu, M., Mizuno, H., Huang, J., Futrell, J.W., et al. (2001) Multilineage cells from human adipose tissue: implications for cell-based therapies. Tissue Engineering, 7, 211-228. doi:10.1089/107632701300062859

[35] Izadpanah, R., Trygg, C., Patel, B., Kriedt, C., Dufour, J., Gimble, J.M. and Bunnell, B.A. (2006) Biologic properties of mesenchymal stem cells derived from bone marrow and adipose tissue. Journal of Cellular Biochemistry, 99, 1285-1297. doi:10.1002/jcb.20904

[36] Lin, T.X., Ambasudhan, R., Yuan, X., Li, W.L., Hilcove, S., et al. (2009) A chemical platform for improved induction of human iPSCs. Nature Methods, 6, 805-808.

[37] Li, W.L., Zhou, H.Y., Abujarour, R., Zhu, S.Y., Joo, Y.J., et al. (2009) Generation of human-induced pluripotent stem cells in the absence of exogenous Sox2. Stem Cells, 27, 2992-3000. doi:10.1002/stem.240

[38] Ichida, J.K., Blanchard, J., Lam, K., Son, E.Y., Chung, J.E., et al. (2009) A small-molecule inhibitor of tgf- $\beta$ signaling replaces Sox 2 in reprogramming by inducing Nanog. Cell Stem Cell, 5, 491-503. doi:10.1016/j.stem.2009.09.012

[39] Esteban, M.A., Wang, T., Qin, B.M., Yang, J.Y., Qin, D.J., et al. (2010) Vitamin C enhances the generation of mouse and human induced pluripotent stem cells. Cell Stem Cell, 6, 71-79. doi:10.1016/j.stem.2009.12.001

[40] Shi, Y., Desponts, C., Do, J.T., Hahm, H.S., Scholer, H.R., et al. (2008) Induction of pluripotent stem cells from mouse embryonic fibroblasts by Oct 4 and Klf4 with smallmolecule compounds. Cell Stem Cell, 3, 568-574. doi:10.1016/j.stem.2008.10.004

[41] Huangfu, D.W., Osafune, K., Maehr, R., Guo, W.J., Eijkelenboom, A., Chen, S.B., Muhlestein, W. and Melton, D.A. (2008) Induction of pluripotent stem cells from primary human fibroblasts with only Oct4 and Sox2. Nature Biotechnology, 26, 1269-1275. 